(C) 2016 IEEE. Personal use of this material is permitted. Permission from IEEE must be obtained for all other uses, in any current or future media, including reprinting/republishing this material for advertising or promotional purposes, creating new collective works, for resale or redistribution to servers or lists, or reuse of any copyrighted component of this work in other works. 


\title{
A Model Predictive Control of Parallel Inverters for Distributed Generations in Microgrids
}

\author{
Mahlagha Mahdavi Aghdam, Student Member, IEEE, Li Li, Member, IEEE, Jianguo Zhu, Senior Member, IEEE \\ Centre of Green Energy and Vehicle Innovation, University of Technology Sydney, Ultimo, NSW 2007, Australia \\ Mahlagha.MahdaviAghdam@student.uts.edu.au
}

\begin{abstract}
Nowadays, microgrid concept has been counted as an effective approach to incorporating distributed renewable resources in distribution networks. Though, the power flow control to share the loads among distributed generations is still an essential focus, specifically under peak demands. This paper focuses on a predictive power flow control method for parallel inverters of distributed generations in microgrids. The microgrid under this case study will operate in the grid-tied mode with appropriate power sharing capability among parallel distributed generations and the main grid. In comparison to the conventional voltage and frequency droop control, the proposed model predictive control approach has shown the better performance, which is verified by the Matlab/Simulink simulation.
\end{abstract}

Keywords-distributed energy resources; grid-tied inverters; microgrids; model predictive control; power flow control;

\section{INTRODUCTION}

Distributed generations (DGs) especially renewable energy sources (RESs) have attracted the market and researchers as practicable alternative in future modern power systems. Most distributed energy resources (DERs) are linked to the grid via an inverter due to its flexibility, good controllability and monitoring capabilities [1]. As the capacities of DGs increase, the inverters connected to them are obligated to perform more proficiently and efficiently to achieve high power quality, stability, and reliability.

Advanced control approaches for power converters have been investigated for a long time in power electronics topics [2]. Also, with the increasing number of inverter-interfaced DERs in advanced power grids, the inverter control has been substantial to achieve the better DER performance. At the microgrid level, these DERs are connected in parallel to the common AC bus. Therefore, to enhance the power quality and maintain the grid stability, it is essential to analyze the power system with parallel inverters.

Various contributions to the parallel arrangement of gridtied inverters are studied in [3-5]. Moreover, various control strategies have been investigated to improve the power system performance through controlling grid-tied inverters.

The droop control technique is a conventional control scheme for controlling the parallel inverters in different applications for example DGs and microgrids [6-8]. This distinguished control method comprises of measuring active and reactive powers, regulating locally and proportionally the output voltage magnitude of each inverter and frequency so as to track the reference values of a system. The droop control methods have the inherent weakness of the slow transient response due to the instantaneous power calculation and the restraint of low pass filter [9].

From the viewpoint of DERs, the inverters ought to be supervised to provide real power to meet the demand and export the surplus into the grid. From the grid point of view, the interfaces would also be capable of providing reactive power, to refine the power quality and boost the stability. Due to this fact, advanced control methods of DERs for power flow optimization are anticipated [10].

In modern industrial controls, the model predictive control (MPC) algorithms have been implemented in many industrial applications [11, 12]. MPC techniques applied to inverters can be considered in two categories: (a) the finite control set MPC (FCS-MPC) approach, which uses the benefit of limited number of switching possibilities of the converter for the optimization problems, and (b) the continuous control set MPC (CCS-MPC) approach, which needs a modulator to produce the switching states from the controller. The FCSMPC has a natural and effective algorithm for controlling power converters, without implementing PI controllers and modulators $[13,14]$. In this paper, we choose the FCS-MPC method for the power flow control in microgrids.

Generally, at each sampling interval, MPC calculates the control optimization problem across a finite prediction window. The optimization of the objectives leads to an optimal control sequence for the entire prediction window, but only the $1^{\text {st }}$ element in the sequence is implemented for the current control. At each sampling instant, this process is repeated with new state estimations or measurements [15].

This paper presents an MPC method for grid-connected parallel inverters in microgrids, which can improve the transient of the power flow in microgrids. The parallel gridtied inverters in a microgrid are described in Section II. The power flow control and power sharing through $V / f$ droop control and MPC are described in Section III. Section IV discusses and compares the results of both control methods through a case study. Finally, Section V is dedicated to the conclusion and future work.

\section{PARALLEL GRID-TIED INVERTERS SYSTEM}

The general architecture of the microgrid is discussed in [16-18]. When connected to the grid, the DGs provide required power to the loads together with the grid. If the generated power of DGs is less than the load consumption, the grid will inject the required power to the load. Alternatively, if 
the generated power of DGs is greater than the load demand, the surplus power from DGs will be fed back to the grid.

Several grid-connected inverters can be linked in parallel to the grid in a way that the sum of each output current of the grid-tied inverter at the point of common coupling (PCC) is equal to the grid current plus the load current as illustrated in Fig 1. This system configuration occurs in microgrids when several DERs are connected to the main grid through a common AC bus.

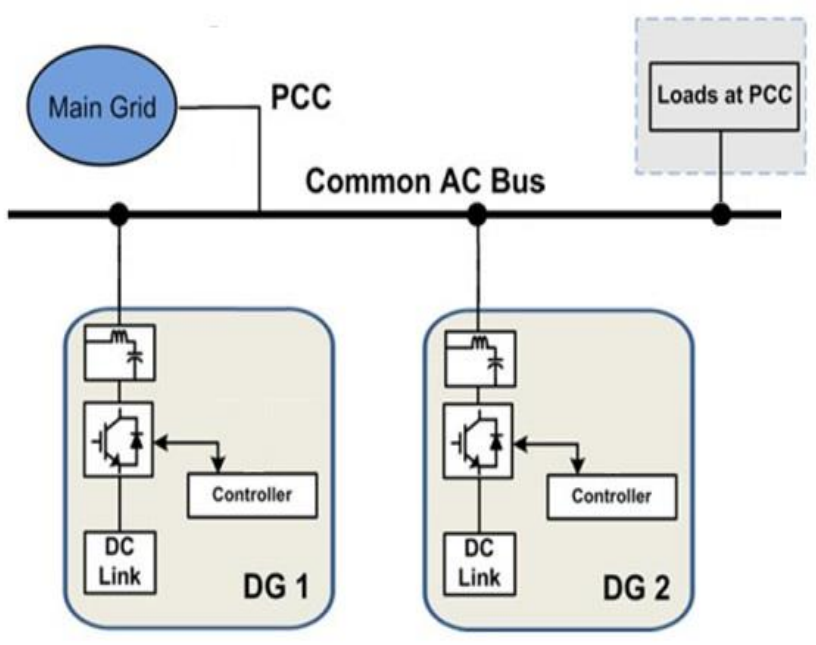

Fig. 1. Parallel inverters in microgrids

The system topology selected for the study involves two parallel inverters. Each of them can perform in the grid-tied mode. A three-phase two-level (3Ph-2L) voltage source inverter (VSI), which is broadly used in industrial applications, has been chosen for each DG. The DC link can be provided by the output of the DER converters or from energy storage units.

The topology of the 3Ph-2L grid-tied inverter consists of three complementary pairs of IGBT power switches. It is connected to the grid via an RL filter and a protection circuit breaker. The total number of achievable switching states is defined as $l=n^{s}$ for an n-phase s-level converter. Thus, eight possible switching states can be found for a $3 \mathrm{Ph}-2 \mathrm{~L}$ VSI. The eight voltage vectors produced by the VSI are expressed by

$$
v_{l}=\left\{\begin{aligned}
\frac{2}{3} e^{j(l-1) \frac{\pi}{3}} V_{d c}, & l & =1, \ldots, 6 \\
0, & l & =0,7
\end{aligned}\right.
$$

The voltage vectors made by each inverter are comprised of six active $(V 1-V 6)$ and two zero $(V 0, V 7)$ voltage vectors, as illustrated in Fig. 2.

\section{POWER FLOW CONTROL FOR LOAD SHARING}

The equivalent circuit of this case study is described in Fig. 3. The complex power flow, $S_{m}=P_{m}+j Q_{m}$ between DGs and the $\mathrm{AC}$ bus can be designated as

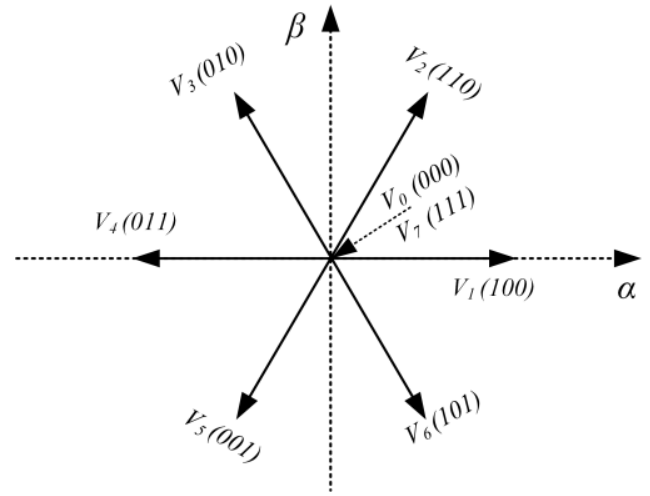

Fig. 2. Possible output voltage vectors for a $3 \mathrm{Ph}-2 \mathrm{~L}$ inverter

$$
\begin{gathered}
P_{m}=\frac{\left|V_{m}\right|^{2} \cos \delta_{m}-\left|V_{m}\right||E| \cos \left(\delta_{m}+\theta_{Z}\right)}{|Z|}, \\
Q_{m}=\frac{\left|V_{m}\right|^{2} \sin \delta_{m}-\left|V_{m}\right||E| \sin \left(\delta_{m}+\theta_{Z}\right)}{|Z|}, \\
\text { for } \quad m=1,2 .
\end{gathered}
$$

where $V_{m}$ and $E$ are the voltage vector of the m-th inverter output and common AC bus, $Z=R+j X=|Z| \angle \theta_{Z}$ is the line impedance, and the power angle $\delta_{m}=\theta_{V_{m}}-\theta_{E}$. The line resistance is usually small and may be neglected, and thus the line impedance will be $Z=j X=|X| \angle 90^{\circ}$.

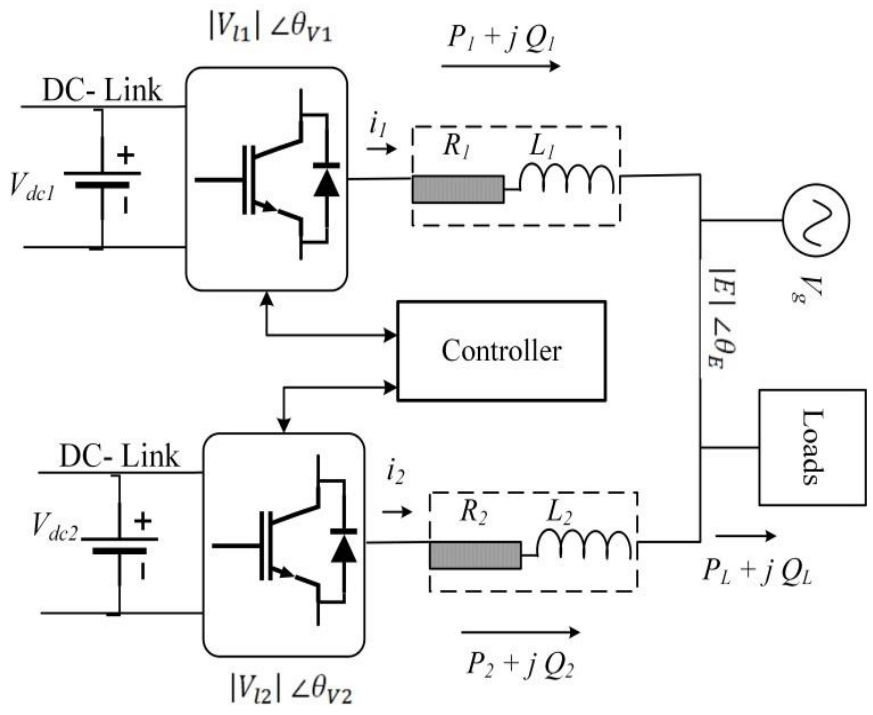

Fig. 3. Equivalent Diagram of the case study

Furthermore, considering that $\delta_{m}$ is generally small, we can adopt $\sin \left(\delta_{m}\right)=\delta_{m}$ and $\cos \left(\delta_{m}\right)=1$, and hence (1) and (2) are expressed as

$$
P_{m}=\frac{\left|V_{m}\right||E| \sin \delta_{m}}{X}=\frac{\left|V_{m}\right||E| \delta_{m}}{X}
$$




$$
Q_{m}=\frac{\left|V_{m}\right|^{2}-\left|V_{m}\right||E| \cos \delta_{m}}{X}=\frac{\left|V_{m}\right|\left(\left|V_{m}\right|-|E|\right)}{X}
$$

Therefore, the active and reactive power flow are dependent on the angle and change in voltage amplitude, respectively.

\section{A. Droop based Active and Reactive Power Flow Control}

Generally, the droop control is used as the conventional control method of parallel inverters for regulating active power and reactive power in microgrids [8]. In this scheme, frequency droop and voltage curves are determined, and the active and reactive power of the DGs can be managed by two PI controllers to regulate the frequency and voltage, correspondingly, based on the pre-defined characteristics. Consequently, the new demands are supported by the DERs which are controlled through the two droop controllers [19].

This approach is based on the power system theory, for example, when the load increases, the frequency is reduced. To this end, the frequency and voltage droop characteristics for microgrids are expressed as

$$
\begin{aligned}
& \omega_{m}-\omega_{r e f}=-g\left(P_{m}-P_{r e f}\right) \\
& V_{m}-V_{\text {ref }}=-h\left(Q_{m}-Q_{r e f}\right)
\end{aligned}
$$

where $P_{\text {ref }}$ and $Q_{\text {ref }}$ are the set point for active and reactive power, $\omega_{\text {ref }}$ and $V_{\text {ref }}$ the set point for frequency and inverter voltage magnitude, and $g$ and $h$ the droop curve slopes. Fig. 4 illustrates the $P$ - $\omega$ and $Q-V$ droop features.

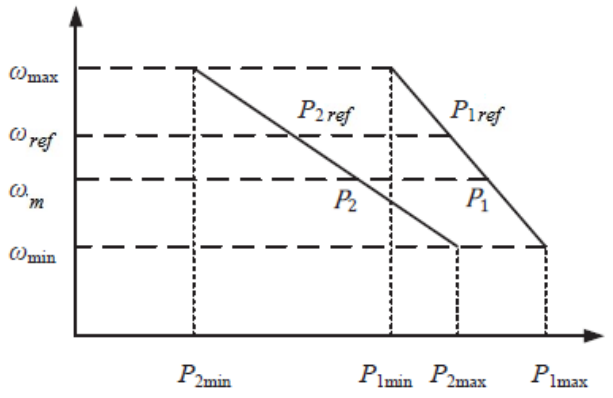

(a)

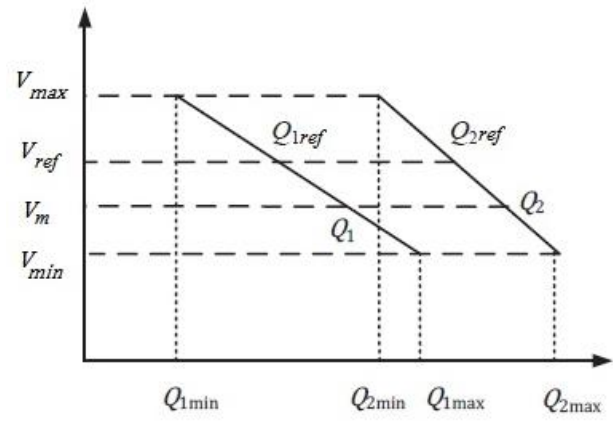

(b)

Fig. 4. Droop characteristics (a) $P-\omega$ (b) $Q-V$
To control the parallel inverters, the instantaneous active and reactive powers are calculated based on the PCC voltage and output current of inverters. The measured powers are then fed to a droop regulator to attain the appropriate power sharing among DGs. In the grid-tied mode, the steady state frequency of the system is set to the grid frequency. The flow chart of $P-\omega$ droop characteristics is depicted in Fig. 5.

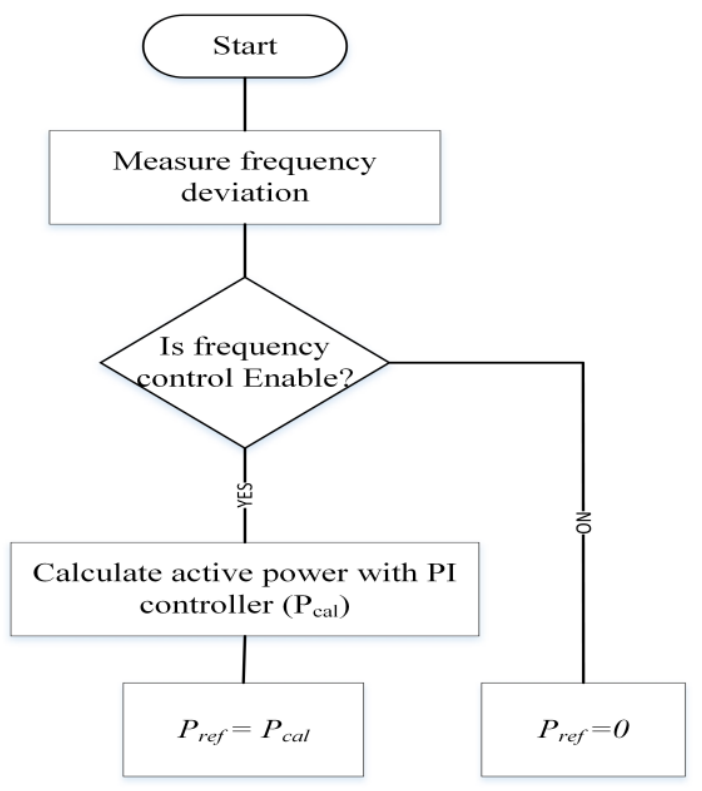

Fig. 5. Flow chart of $P-\omega$ droop characteristics

\section{B. MPC-based Direct Power Control}

To help improve the power quality and enhance the system stability, adaptable active and reactive power regulation has to be gained. Hence, the FCS-MPC is recommended to regulate the active/reactive power flow. The MPC chooses the optimal voltage vector sequence for each inverter to meet the load demand and in the meantime maintain the power flow at a predetermined level between the microgrid to main grid. The FCS-MPC takes benefits of the discrete characteristic of power converters in the MPC calculations with reduced computation time. As there are finite numbers of switching states in each inverter, the prediction procedure will be conducted through minimizing the cost function.

\section{1) Modeling of the System}

This strategy, direct power control (DPC), involves a predictive model of the instantaneous power evolution. In the fixed reference frame $\alpha-\beta$ and for a symmetrical threephase power system, instantaneous active and reactive powers by grid-tied inverters can be derived below.

Note that

$$
\begin{gathered}
i_{m \alpha \beta}(k+1)=i_{m \alpha \beta}(k)+\frac{T_{s}}{L}\left[V_{m \alpha \beta}^{\left(l_{m}\right)}(k)-V_{g \alpha \beta}(k)\right. \\
\left.-R i_{m \alpha \beta}(k)\right]
\end{gathered}
$$

$$
\text { for } m=1,2 \text { and } l_{m}=0,1, \ldots, 7 \text {. }
$$


where $m$ is the number of inverters connected in parallel to the common bus, and $l_{m}$ denotes the possible switching state of the $m$-th inverter. The predicted current to the grid can be expressed as

$$
\begin{gathered}
i_{o \alpha \beta}(k+1)=i_{m \alpha \beta}^{\left(l_{1}\right)}(k+1)+i_{m \alpha \beta}^{\left(l_{2}\right)}(k+1) \\
-i_{L \alpha \beta}(k+1)
\end{gathered}
$$

Available active and reactive powers to the grid are defined as

$$
\begin{gathered}
P(k)=\frac{3}{2}\left[V_{g \alpha} i_{o \alpha}(k)+V_{g \beta} i_{o \beta}(k)\right] \\
Q(k)=\frac{3}{2}\left[-V_{g \beta} i_{o \alpha}(k)+V_{g \alpha} i_{o \beta}(k)\right]
\end{gathered}
$$

\section{2) Cost Function}

Define the power generated by the grid-connected DG systems as $P_{D G}$, and the power demand by loads as $P_{\text {demand }}$. The net power to the main grid can be obtained by

$$
\begin{gathered}
P_{n e t}=P_{D G}-P_{\text {demand }} \\
P_{D G}=P_{1}+P_{2}
\end{gathered}
$$

Thus, the predicted power to the grid can be gained as the following

$$
\begin{aligned}
P_{n e t}(k+1) & =\frac{3}{2}\left[V_{g \alpha} i_{o \alpha}(k+1)+V_{g \beta} i_{o \beta}(k+1)\right] \\
Q_{n e t}(k+1) & =\frac{3}{2}\left[-V_{g \beta} i_{o \alpha}(k+1)+V_{g \alpha} i_{o \beta}(k+1)\right]
\end{aligned}
$$

The current and voltage available to the grid are measured at the current sampling time and applied to (7-11) to compute $P_{\text {net }}$ and $Q_{\text {net }}$ at the next sampling time for all switching states of each inverter. The cost function is defined as

$$
J=\left[P_{\text {ref }}-P_{n e t}(k+1)\right]^{2}+\left[Q_{\text {ref }}-Q_{n e t}(k+1)\right]^{2}
$$

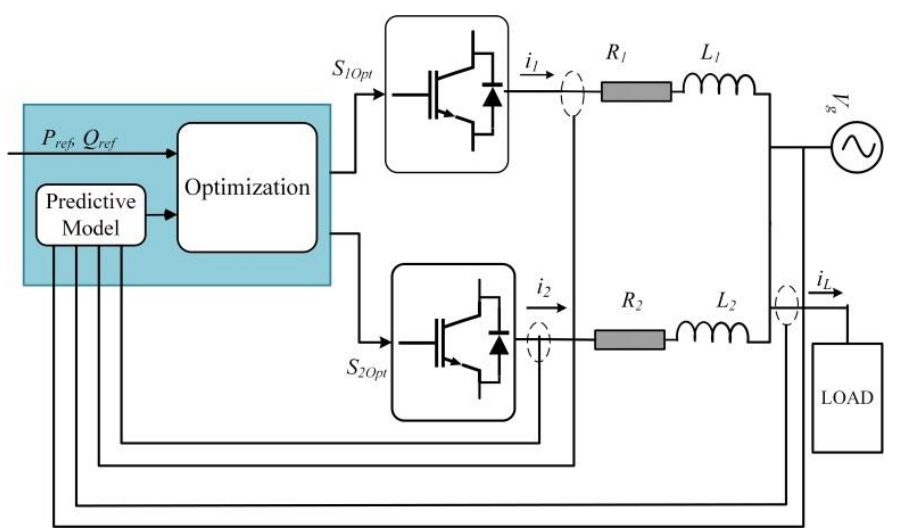

Fig. 6. Scheme diagram of the system with MPC controller

The minimization of the cost function leads to an optimal power flow of two DGs for the transmitted power to the grid at the scheduled reference level. The scheme diagram of the suggested algorithm is illustrated in Fig. 6.

\section{SIMULATION}

\section{A. Simulation Results and Discussion}

Simulation of two parallel 3P-2L inverters in the grid-tied mode in a microgrid application shown in Fig. 2 is executed by using the MATLAB/Simulink. Table 1 tablets the system parameters for this case study. The active and reactive powers are both initially set to zero. The active power reference varies from 0 to $-4 \mathrm{~kW}$ at $0.05 \mathrm{~s}, 0 \mathrm{~W}$ at $0.06 \mathrm{~s}, 2 \mathrm{~kW}$ at $0.07 \mathrm{~s}$, and $0 \mathrm{~W}$ at $0.08 \mathrm{~s}$, and maintains $0 \mathrm{~kW}$, whereas the reactive power reference is altered from 0 to $-3,0$, and $1 \mathrm{kVAr}$ at 0.02 s, $0.04 \mathrm{~s}$, and $0.09 \mathrm{~s}$, respectively.

\begin{tabular}{lcc}
\multicolumn{3}{c}{ TABLE I. System Parameters } \\
\hline Grid voltage & $V_{g}$ & $100 \mathrm{~V}$ \\
\hline Load active power & $P_{L}$ & $3 \mathrm{~kW}$ \\
Voltage frequency & $f$ & $50 \mathrm{~Hz}$ \\
\hline Sampling period & $T_{s}$ & $50 \mu \mathrm{s}$ \\
& DG1 \\
\hline Filter resistance & $R_{1}$ & $0.51 \Omega$ \\
\hline Filter inductance & $L_{1}$ & $4.8 \mathrm{mH}$ \\
\hline Dc source voltage & $V_{d c 1}$ & $250 \mathrm{~V}$ \\
& DG2 & $1 \Omega$ \\
\hline Filter resistance & $R_{2}$ & $10 \mathrm{mH}$ \\
\hline Filter inductance & $L_{2}$ & $400 \mathrm{~V}$ \\
\hline Dc source voltage & $V_{d c 2}$ & \\
\hline
\end{tabular}

Fig. 7 illustrates the performance of V/f droop control approach for power flow control. Figs. 8 and 9 illustrate the performance of MPC controller in the cases of individual DG and parallel DGs respectively. It is shown that the suggested FCS-MPC method presents much better power flow tracking. The current and voltage of the common bus are demonstrated in Fig. 10. As observed, the voltage and current are sinusoidal, so the system performance is stable.
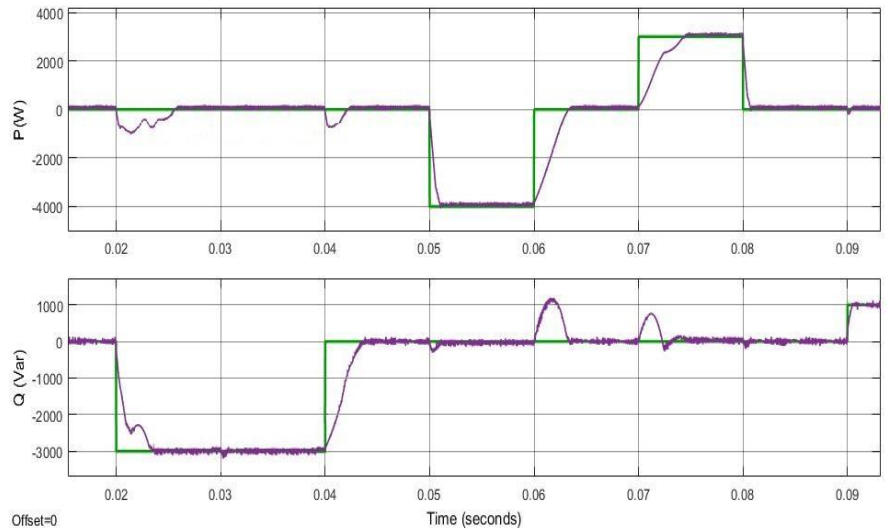

Fig. 7. Active and reactive power flow - Droop control

\section{B. Comparison}

The DGs that are connected in parallel can take the appropriate actions in response of changes of the load demand.The controller tracks the load demand to maintain the net active/reactive power flow into the main grid at the scheduled level. 

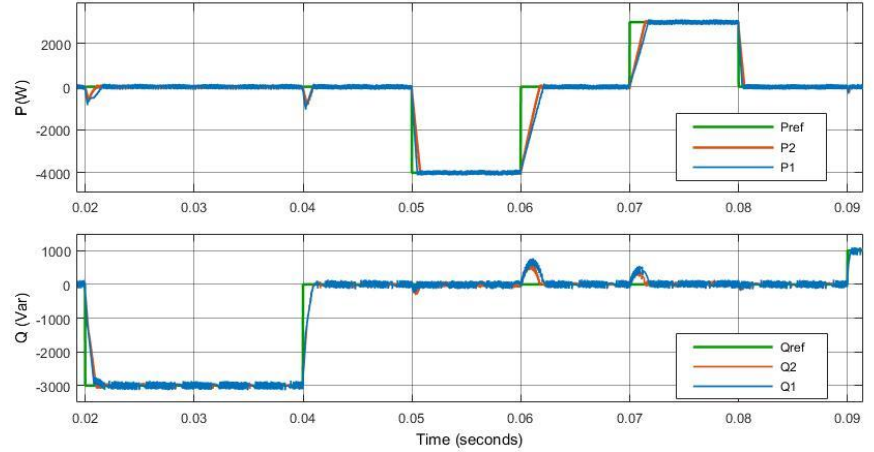

Fig. 8. Power flow control for individual DG - MPC
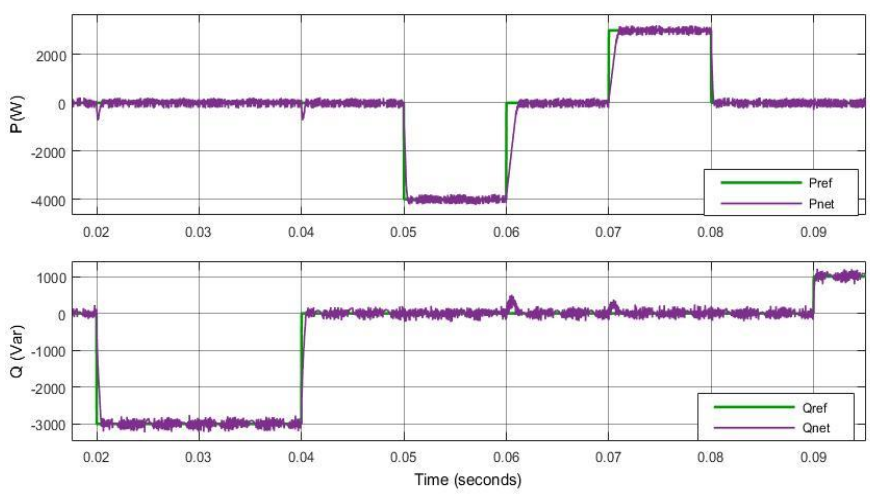

Fig. 9. Power flow control for net power to the grid - MPC
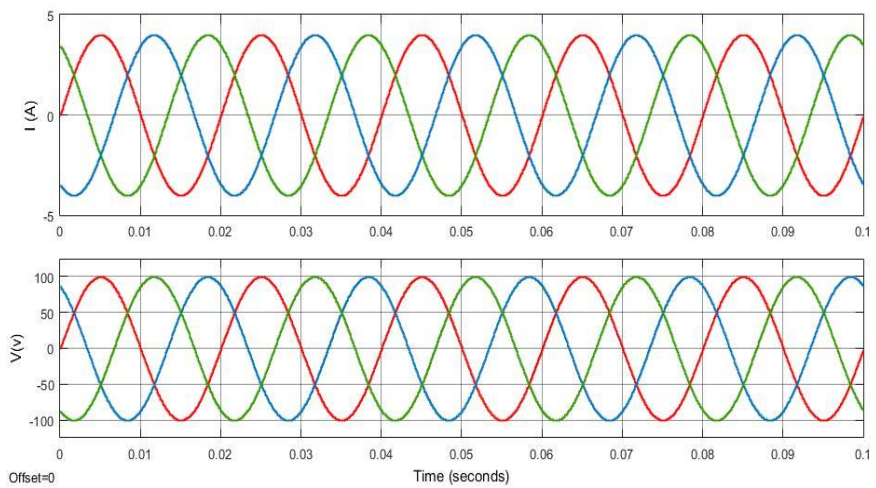

Fig. 10. The current and voltage of the AC common bus - MPC

As can be seen in Figs. 8 and 9, the MPC approach shows a great performance and power flow tracking, in comparison to the droop control scheme. As aforementioned, the droop control as a conventional method for power sharing between parallel connected DERs has an inherent flaw of slow transient response due to the powers calculation and the restraint of low pass filter.

Table II tabulates a quantitative comparison of indicators, including the frequency, power ripples, rise time, and settling time, between the droop control and the MPC methods by simulation in MATLAB/Simulink.
It can be seen in Figs. 7 and 9, the MPC algorithm shows much better performance than the droop control in term of power sharing between two parallel grid-connected inverters. The measurements have shown that although the active and reactive power ripples have not been compensated much by the MPC approach, the rise time and settling time are reduced considerably. As a result, the MPC scheme provides a better transient dynamics than the droop control scheme.

TABLE II. Quantitative Comparison

\begin{tabular}{|c|c|c|c|c|c|}
\hline $\begin{array}{l}\text { Power } \\
\text { Sharing }\end{array}$ & $f_{s}(\mathrm{~Hz})$ & $P_{\text {rip }}(W)$ & $Q_{\text {rip }}($ Var $)$ & $\begin{array}{l}\text { Rise time } \\
(\mu \mathrm{Sec})\end{array}$ & $\begin{array}{c}\text { Settling } \\
\text { time } \\
(\mathrm{mSec})\end{array}$ \\
\hline V/f droop & $20 \mathrm{k}$ & 158.5 & 269.87 & 1408.10 & 645.588 \\
\hline MPC (DG1) & $20 \mathrm{k}$ & 176.24 & 485 & 793.601 & 60.810 \\
\hline MPC (DG2) & $20 \mathrm{k}$ & 195.43 & 527 & 793.601 & 58.109 \\
\hline $\begin{array}{c}\text { MPC } \\
\text { (Parallel) }\end{array}$ & $20 \mathrm{k}$ & 126.8 & 215.8 & 793.601 & 20.023 \\
\hline
\end{tabular}

\section{CONCLUSION AND FUTURE WORK}

In this work, an MPC scheme is employed for the power sharing problem of parallel DGs in a microgrid. The equivalent microgrid model is established and the associated cost function is formulated. The case study has been considered and the results show that the MPC technique attains better performance on the power flow control than the conventional droop control scheme. Furthermore, the DGs can respond appropriately to the load changes such that the power flow to the main grid is maintained at the scheduled level. The MPC approach is compared with the conventional frequency and voltage droop control approach in this case study.

For the future work, improving power quality and enhancing the system stability for both the islanded mode and gird-connected mode will be investigated. Moreover, seamless switching between two modes is an important concern that needs to be considered in the controller design to achieve power sharing without producing undesirable impacts on the grid and critical loads.

\section{REFERENCES}

I. Colak, E. Kabalci, G. Fulli, and S. Lazarou, "A survey on the contributions of power electronics to smart grid systems," Renewable and Sustainable Energy Reviews, vol. 47, pp. 562-579, 2015.

[2] S. Vazquez, A. Marquez, R. Aguilera, D. Quevedo, J. I. Leon, and L. G. Franquelo, "Predictive optimal switching sequence direct power control for grid-connected power converters," Industrial Electronics, IEEE Transactions on, vol. 62, pp. 2010-2020, 2015.

[3] M. N. Arafat, A. Elrayyah, and Y. Sozer, "An Effective Smooth Transition Control Strategy Using Droop-Based Synchronization for Parallel Inverters," Industry Applications, IEEE Transactions on, vol. 51, pp. 2443-2454, 2015.

[4] J. He, Y. Li, D. Bosnjak, and B. Harris, "Investigation and active damping of multiple resonances in a parallel-inverter-based microgrid," Power Electronics, IEEE Transactions on, vol. 28, pp. 234-246, 2013.

[5] W. Choi;, W. Lee;, and B. Sarlioglu;, "Effect of grid inductance on grid current quality of parallel grid-connected inverter system with output LCL filter and closed-loop control," presented at the 2016 IEEE Applied Power Electronics Conference and Exposition (APEC), 2016. 
[6] M. Dehghani, M. H. Khooban, T. Niknam, and S. Rafiei, "TimeVarying Sliding Mode Control Strategy for Multibus Low-Voltage Microgrids with Parallel Connected Renewable Power Sources in Islanding Mode," Journal of Energy Engineering, p. 05016002, 2016.

[7] J. He and Y. W. Li, "An enhanced microgrid load demand sharing strategy," Power Electronics, IEEE Transactions on, vol. 27, pp. 3984-3995, 2012.

[8] V. Nasirian, Q. Shafiee, J. M. Guerrero, F. L. Lewis, and A. Davoudi, "Droop-free distributed control for ac microgrids," Power Electronics, IEEE Transactions on, vol. 31, pp. 1600-1617, 2016.

[9] Z. Shuai, Y. Sun, Z. J. Shen, W. Tian, C. Tu, Y. Li, et al., "Microgrid stability: Classification and a review," Renewable and Sustainable Energy Reviews, vol. 58, pp. 167-179, 2016.

[10] J. Hu, J. Zhu, and J. M. Guerrero, "Model predictive control of smart microgrids," in Electrical Machines and Systems (ICEMS), 2014 17th International Conference on, 2014, pp. 2815-2820.

[11] T. Geyer and D. E. Quevedo, "Performance of multistep finite control set model predictive control for power electronics," Power Electronics, IEEE Transactions on, vol. 30, pp. 1633-1644, 2015.

[12] D. Quevedo and T. Geyer, "Model predictive control with reference tracking," ed: Google Patents, 2015.

[13] M. Mahdavi Aghdam, L. Li, J. Zhu, and O. Palizban, "Finite Control Set Model Predictive Control-A Powerful Control Algorithm for Grid-Connected Power Converters," in Industrial
Electronics and Applications (ICIEA), 2016 11th IEEE Conference on, Hefei, China, 2016.

[14] J. Rodriguez, M. P. Kazmierkowski, J. R. Espinoza, P. Zanchetta, H. Abu-Rub, H. A. Young, et al., "State of the art of finite control set model predictive control in power electronics," Industrial Informatics, IEEE Transactions on, vol. 9, pp. 1003-1016, 2013

[15] J. Hu, J. Zhu, and D. G. Dorrell, "Model predictive control of gridconnected inverters for PV systems with flexible power regulation and switching frequency reduction," Industry Applications, IEEE Transactions on, vol. 51, pp. 587-594, 2015.

[16] A. H. Etemadi, E. J. Davison, and R. Iravani, "A decentralized robust control strategy for multi-DER microgrids-Part I: Fundamental concepts," Power Delivery, IEEE Transactions on, vol. 27, pp. 1843-1853, 2012.

[17] N. Lidula and A. Rajapakse, "Microgrids research: A review of experimental microgrids and test systems," Renewable and Sustainable Energy Reviews, vol. 15, pp. 186-202, 2011.

[18] C. Marnay and G. Venkataramanan, "Microgrids in the evolving electricity generation and delivery infrastructure," in Power Engineering Society General Meeting, 2006. IEEE, 2006, p. 5 pp.

[19] J. C. Vasquez, J. M. Guerrero, M. Savaghebi, J. Eloy-Garcia, and R. Teodorescu, "Modeling, analysis, and design of stationaryreference-frame droop-controlled parallel three-phase voltage source inverters," Industrial Electronics, IEEE Transactions on, vol. 60, pp. 1271-1280, 2013. 